\title{
Runaway electron beam stability and decay in COMPASS
}

O. Ficker ${ }^{1,2}$, E. Macusova ${ }^{1}$, J. Mlynar ${ }^{1}$, D. Bren ${ }^{2}$, A. Casolari ${ }^{1}$, J. Cerovsky ${ }^{1,2}$, M. Farnik ${ }^{1,2}$, O. Grover ${ }^{1,2}$, J. Havlicek ${ }^{1}$, A.

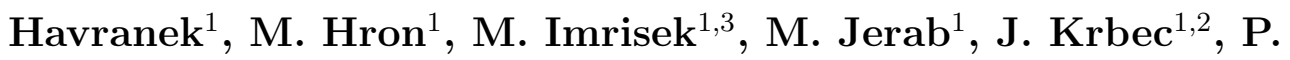
Kulhanek $^{1}$, V. Linhart ${ }^{2}$, M. Marcisovsky ${ }^{2}$, T. Markovic ${ }^{1,3}$, D.

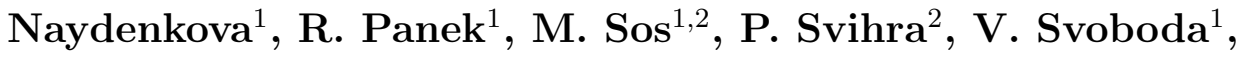
M. Tomes ${ }^{1,3}$, J. Urban ${ }^{1}$, J. Varju ${ }^{1}$, M. Vlainic ${ }^{4}$, P. Vondracek ${ }^{1,3}$, V. Vrba ${ }^{2}$, V. Weinzettl ${ }^{1}$, D. Carnevale ${ }^{5}$, J. Decker ${ }^{6}$, M.

Gobbin $^{7}$, M. Gospodarczyk ${ }^{5}$, G. Papp ${ }^{8}$, Y. Peysson ${ }^{9}$, V.V. Plyusnin $^{10}$, M. Rabinski ${ }^{11}$, C. Reux ${ }^{9}$, COMPASS team ${ }^{1}$ and the EUROfusion MST1 Team*

${ }^{1}$ Institute of Plasma Physics of the CAS, CZ-18200 Praha 8, Czech Republic

2 FNSPE, Czech Technical University in Prague, CZ-11519 Praha 1, Czech Republic

${ }^{3}$ FMP, Charles University, Ke Karlovu 3, CZ-12116 Praha 2, Czech Republic

${ }^{4}$ Institute of Physics, University of Belgrade, 11080 Belgrade, Serbia

${ }^{5}$ Universita' di Roma Tor Vergata, 00133 Roma, Italy

${ }^{6}$ Swiss Plasma Centre, EPFL, CH-1015 Lausanne, Switzerland

7 Consorzio RFX, Corso Stati Uniti 4, 35127 Padova, Italy

8 Max Planck Institute for Plasma Physics, Garching D-85748, Germany

${ }^{9}$ CEA, IRFM, F-13108 Saint-Paul-lez-Durance, France

${ }^{10}$ Instituto de Plasmas e Fusao Nuclear, IST, Lisbon, Portugal

${ }^{11}$ National Centre for Nuclear Research (NCBJ), Otwock-Swierk, Poland

* See the author list "H. Meyer et al 2017 Nucl. Fusion 57 102014"

E-mail: ficker@ipp.cas.cz

Abstract. The paper presents two scenarios used for generation of runaway electron (RE) beam in the COMPASS tokamak with a focus on the decay phase and control of the beam. The first scenario consists of argon massive gas injection (MGI) into the current ramp-up phase leading to a disruption accompanied by runaway plateau generation. In the second scenario, injection of smaller amount of gas is used in order to isolate runaway electron beam from high temperature plasma. The current control and radial and vertical position feedback control performances in the second scenario were experimentally studied and analysed. The role of $\mathrm{RE}$ energy in the radial position stability of the RE beam seems to be crucial. A comparison of the decay phase of RE beam in various amounts of Ar or Ne was studied using AXUV (absolute extreme ultraviolet) tomography and HXR (hard X-ray) intensity measurement. Argon clearly leads to higher HXR fluxes for the same current decay rate compared to neon, while radiated power based on AXUV measurements is larger for $\mathrm{Ne}$ in the same set of discharges. 
Keywords: tokamaks, runaway electrons, MHD instabilities, disruptions

Submitted to: Nucl. Fusion

\section{Introduction}

Runaway electrons have been extensively studied at COMPASS within the framework of EUROfusion MST1 (Medium Size Tokamaks) work package as they still present an issue with respect to the safe operation of ITER [1. Without securing mitigated disruptions with no RE being generated and/or without developing a fully reliable runaway beam mitigation technique, ITER can hardly succeed. It seems that the typical timescales of disruptions in ITER will be crucial for the beam generation 2] and that the position stability in the post-disruptive phase remains the critical issue [3]. Enough time to mitigate the RE beam can be only secured in case that the beam position is stabilised which may be extremely difficult. Alternatively, the speed of the position instability must be known to optimise the mitigation method. In ITER, it is expected that the shaping field may cause the vertical instability of the beam just after the disruption while correct stabilizing vertical field can be hardly optimised early enough to secure also the radial position stability. However, on currently operated machines, the stability of the beam can be achieved and various mitigation techniques, including injection of large amounts of noble gases or shattered pellets [4, can be studied under controlled conditions. In fact, if the position control is reliable enough, the amount of gas already present in the chamber from the disruption mitigation may be sufficient to slowly mitigate the beam. Detailed understanding of beam position stability behaviour under different conditions may bring a useful information to ITER. The control and mitigation of the RE beam is one of the key topics in European fusion research, see [5],[6] and also [7].

\subsection{COMPASS and RE experiments}

The COMPASS tokamak is one of the European compact, highly flexible facilities that has been operated at IPP Prague since 2008. The vacuum chamber is D-shaped with an open divertor. The major radius is $R_{0}=0.56 \mathrm{~m}$ and the minor radius is $a=0.21 \mathrm{~m}$ . The toroidal magnetic field $B_{t}$ ranges from 0.8 to $1.6 \mathrm{~T}$ while plasma current $I_{p}$ up to $350 \mathrm{kA}$ can be driven in the tokamak. The machine is equipped with two $40 \mathrm{keV}$ NBIs with heating power up to $350 \mathrm{~kW}$ and capable of routine H-mode operation [8], [9]. The $\mathrm{RE}$ research on COMPASS gains from the machine flexibility, increasing experience in RE experiments at EUROfusion MST1 machines and cooperation with groups working on relevant diagnostics development and models.

During the COMPASS RE experiments, the RE generation in quiescent scenarios and losses related to various MHD (magnetohydrodynamics) phenomena and external field errors were studied as well as massive gas injection (MGI) triggered disruptions in 
the ramp-up scenario [10],[11]. In the flattop discharges without gas injection the losses of $\mathrm{RE}$ are modified to a large extent by sawtooth instability, magnetic island rotation and poloidal field power supply oscillations (introduced by flywheel rotation and set of 12-pulse convertors) [12. The reaction of REs to the perturbed magnetic fields was recognized as an important topic. Thanks to the rich variety of possible resonant magnetic perturbation (RMP) coil configurations at COMPASS it is possible to run very detailed scans of the RMP effect on $\mathrm{RE}$ beam. So far, $n=1$ and $n=2$ low field side (LFS) off-midplane coil configurations were tested with promising results [13, [14] and good agreement with the results achieved at ASDEX-U [15]. Despite the fact that ITER ELM (edge localised mode) control coils are probably unable to affect the RE orbits in the confined RE beam as they are too far from the plasma, the COMPASS experiments can contribute to the understanding of the beam behaviour in the perturbed fields and to the validation of theoretical predictions and models of runaway electron transport in perturbed fields. The most urgent task of the RE research is to find an effective RE beam mitigation method that can be reliably extrapolated to ITER. The unmitigated RE beam termination may occur on ab order of magnitude shorter time scales than a typical plasma disruption. At COMPASS, terminations of extremely low-density plasmas in the slide-away regime on microsecond time scales due to radial position instability were recorded [13]. These sudden terminations also caused very localised hotspots. On the other hand, after injection of even a minor amount of high-Z impurity gas the current decay has been rather moderate and no severe hotspots were observed.

\subsection{Diagnostics and gas injection valves}

COMPASS is equipped with a rich set of magnetic diagnostics [16] which contributes not only to equilibrium reconstruction and control of the discharge parameters but also to the study of the magnetic fluctuations related to various instabilities. The line-averaged density is determined and controlled using a $2 \mathrm{~mm}$ microwave interferometer, the density and temperature profiles are measured using Thomson Scattering (TS) system [17]. The detection of REs is carried out using multiple methods, the low energy RE (about $100 \mathrm{keV}$ ) confined in the vessel volume are detected using a vertical ECE (Electron Cyclotron Emission) system [18] and the lost RE can be detected by the Cherenkov detector [19], 20], but namely using several HXR detectors measuring the secondary radiation (unshielded and shielded with HXR energy thresholds roughly $E>50 \mathrm{keV}$ and $E>500 \mathrm{keV}$ ). The photo-neutrons may be also detected by multiple neutron detectors, however the contribution of photo-neutrons is mixed with HXR in case of large fluxes that affect even the shielded detectors. The wide-angle view and detailed observation of the RE beam in the visible range is provided by fast cameras Photron MINI UX-100 [21] and Photron SA-X2, respectively. Spectral data in visible, near IR and near UV regions are acquired using various mini-spectrometers. To some degree, SXR (soft X-ray) cameras and AXUV cameras may also contribute to the analysis of 
RE experiments, despite the fact that they can be affected by HXR radiation from the walls, see section 1.3 . The gas injection experiments use two very different valves: (i) the ex-vessel piezoelectric valve injecting smaller amount of gas from the high field side (HFS) divertor region (also used for seeding experiments), can inject gas atoms at rates roughly $2 \cdot 10^{20} \mathrm{~s}^{-1}$ when used with $\mathrm{Ar}$ and pressures around 1 bar. With standard opening time $20 \mathrm{~ms}$, this gives $4-5 \cdot 10^{18}$ injected gas particles according to the calibration; (ii) the ex-vessel MGI solenoid valves at 3 different toroidal positions at outer mid-plane can injected at rates roughly $1 \cdot 10^{22} \mathrm{~s}^{-1}$ when used with Ar and at pressure 2 bars, while pressure up to 5 bars can be used. As typical opening times of the valve close to $10 \mathrm{~ms}$, the amount of injected gas particles is up to $1 \cdot 10^{20}$.

\subsection{Modified tomographic inversion}

The hard X-ray radiation affecting the LFS angular cameras during runaway electron beam scenarios causes an unknown contribution to the SXR and AXUV signals and a special modified procedure needs to be used for the tomography reconstruction. The bottom HFS AXUV camera F (for field of view see figure 1) is the least affected one, providing peaked profiles of radiation even during high energy and high current $\mathrm{RE}$ beams. It provides radial resolution, however use of a single camera in the unconstrained Minimum Fisher Regularisation (MFR) tomography (for application on COMPASS see [22]) would cause a vertically spread artefacts. On the other hand, application of Abelian inversion is too dependent on the use of magnetic equilibrium data, which is not sufficiently correct for the RE beam. Therefore, MFR using smoothing given by gradient map of the $\Psi(R, z)$ function [23] and modified reconstruction domain (circular area on the mid-plane) was developed and used to obtain the radiation patterns and approximate (due to non-uniform AXUV spectral response) radiated power values. This treatment helps to avoid both the artefacts and the contribution from the limiter radiation. An example of the used smoothing functions and a typical axis-peaked radiation profile with low intensity halo during the RE beam phase are shown in figure 1 .

\section{Runaway electron beam scenarios}

\subsection{Ramp-up scenario}

COMPASS is characterised by relatively low toroidal magnetic field which should not be beneficial for the post-disruptive RE beam generation as was shown, e.g. in TEXTOR [24. On the other hand, RE beams in the ramp-up phase following a classical disruption triggered by massive gas injection (fast thermal quench (TQ), often current spike, current quench (CQ) and beam plateau) were achieved irregularly, see an example discharge in figure 2 where $B_{t}=1.15 \mathrm{~T}$. The scenario includes a carefully tuned fuelling waveform, injection at early times (low currents/high $q_{95}$ ), optimised position reference and argon MGI (see section 1.2 for the description of the valve) at pressure 1-3 bars and short valve opening time. Despite the relatively low reproducibility, a systematic 

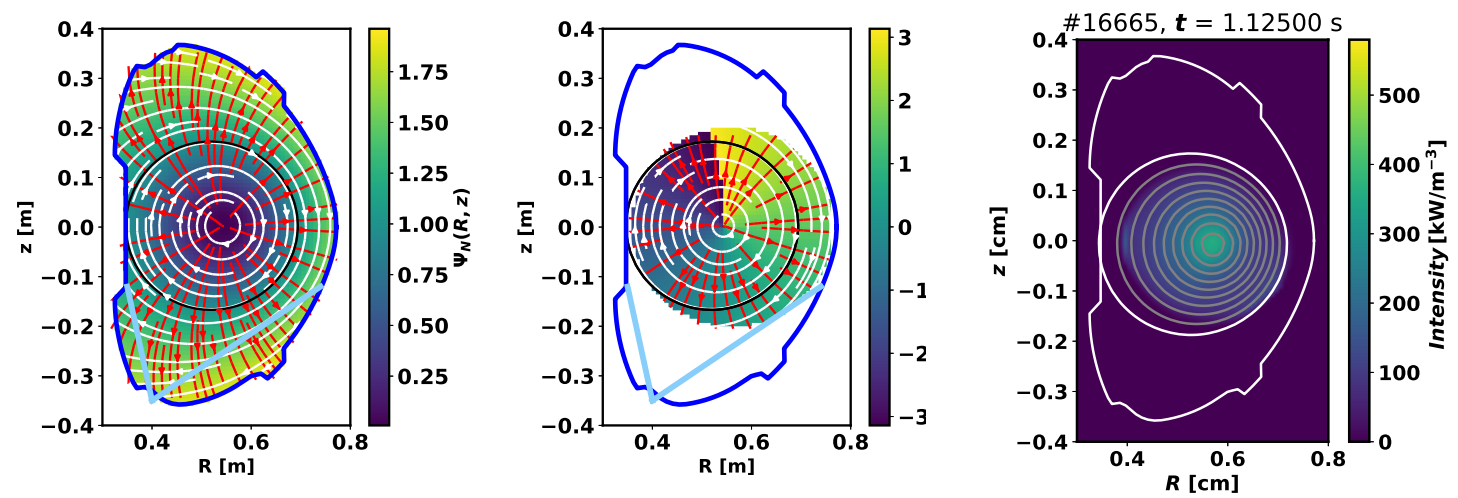

Figure 1. Tomography procedure for reduced set of LOS: left: COMPASS vessel with field of view of AXUV camera F (light blue lines) marked over the $\Psi(R, z)$ map, center: modified reconstruction domain with preferential smoothing direction parameter - ratio of $\nabla \Psi$ components with respect to the flux contours, left: typical radiation pattern of an evolved RE beam from the flattop Ar gas puff scan described further in the text.

analysis brought valuable results [10],[11]. Recently the crucial role of the toroidal magnetic field has been confirmed in a dedicated scan [13. No RE beams were created in the very same scenario at fields lower than $1.1 \mathrm{~T}$ although the beam was reliably produced at higher fields. Based on magnetic measurements and on AXUV inversion and camera data, it is concluded that the discharges, where Ar MGI does not lead to RE beam generation, terminate on the HFS. In contrast, the discharges where beam is generated following the current quench, the radiation pattern shrinks to vicinity of the vessel axis during the TQ and CQ [25]. In this case, the low energy channel of the Cherenkov detector $(E>57 \mathrm{keV})$ indicates fast electron population existence already in the phase of current quench. These beams are often characterised by a very interesting first stage, where bright filaments in the camera images correlate with the short spikes in the ECE and Mirnov coil data and later also in the HXR and Cherenkov detector data [26]. Typically, the generated RE beams are radially unstable - the instability is more severe in the case of a smaller beam current, i.e. larger drop of current during the current quench. Well confined beams expand in the major radius and are lost to the LFS, or partially lost and then stabilised as in the case of the discharge in figure 2 .

\subsection{Flattop scenario}

Due to the low reproducibility of ramp-up scenario which would be problematic namely in the RE beam decay experiments and scans, an alternative, more quiescent scenario was developed. In these discharges, the current flattop is reached and the fuelling is turned off which leads to the decay of the thermal plasma density and a rise of the $\mathrm{RE}$ current fraction in several tens of ms. Ar or Ne injection is then introduced using piezoelectric gas puff or MGI (see section 1.2). MGI causes a significantly faster decay of the RE current, see [13]. Within a short delay (5 ms) after the injection when the gas fills the poloidal cross-section, the derivative of the current in the primary windings (which 

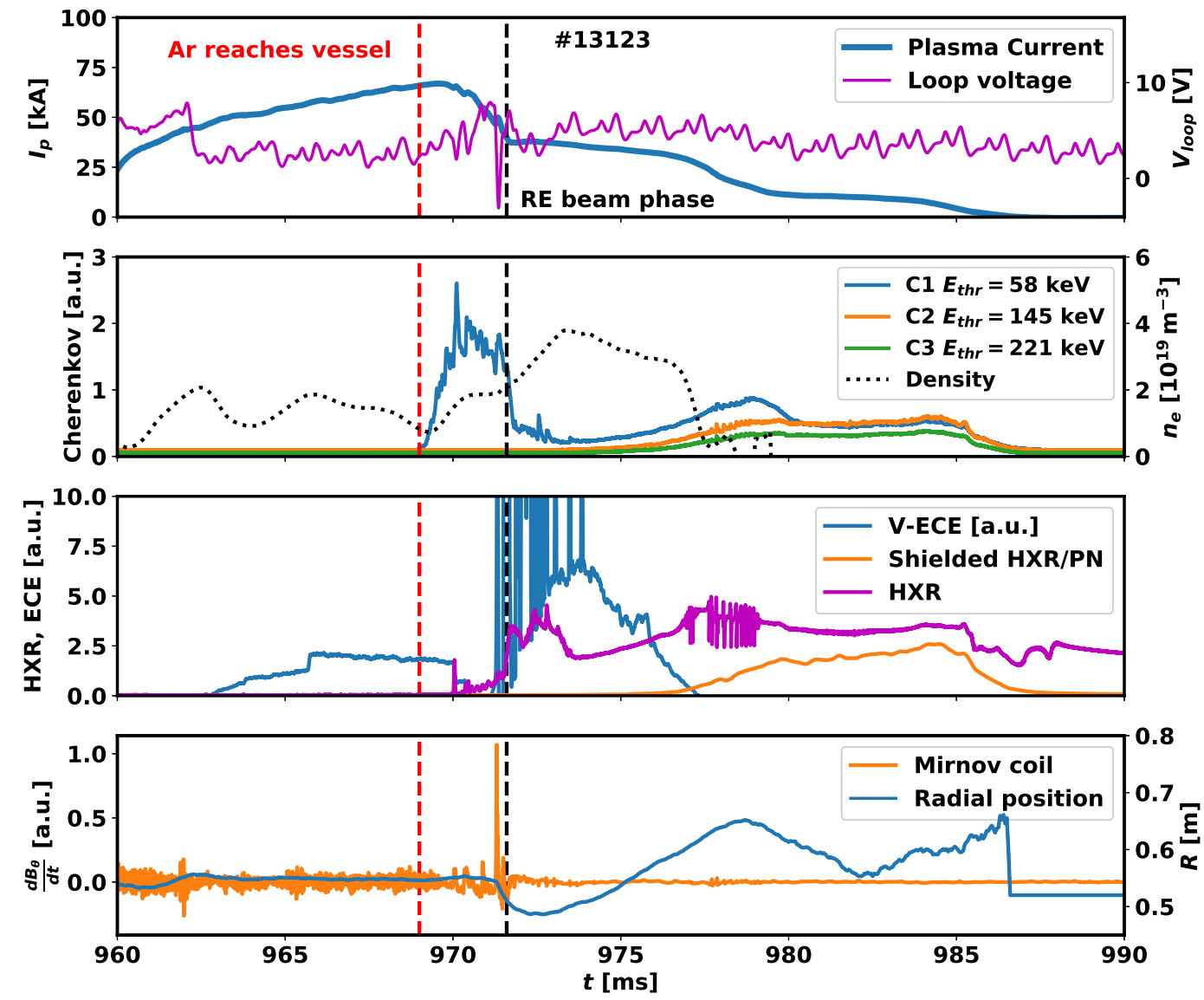

Figure 2. The ramp-up scenario for RE beam generation: Plasma current ramp-up is interrupted by Ar MGI which leads to a current quench and RE generation. The density (black dashed line) increases, and the early beam phase is accompanied by spikes in the ECE data and increased level of HXR. Later the signals of higher-energy Cherenkov detector channels and shielded HXR detector increase as well

creates the external loop voltage) is set to zero, see figure 3 for the detailed overview. During this stage, additional puffs or RMPs [13,,14] may be applied to investigate the influence on the decay of the beam. The puff causes the quench of the thermal plasma while the REs are preserved almost unaffected and fully overtake the remaining current. The TQ is very slow in the case of piezo gas-puff lasting roughly $5 \mathrm{~ms}$ (see the profile $T_{e}$ evolution in figure 4) while in the case of MGI, TQ lasts less than $1 \mathrm{~ms}$. The amount and time of the gas injection plays a crucial role. While this scenario reliably works with the piezoelectric valve injection (slow TQ) at almost any time during the low density discharge, including the late phase of the ramp-up, the MGI typically causes immediate current quench when injected too early, while RE beam generation and gradual beam decay is the result of the later injection, see figure 4. This indicates that the slower TQ 
allows to reach sufficient RE energy and/or current during the injection itself while the fast MGI may only preserve the beam in the case that the RE population is already well evolved. All the results described further in this manuscript are based on this scenario. Advantages and possible applications of the flattop scenario:

- Reproducible conditions - suitable for scans (e.g. $n_{e}, B, \mathrm{RMP}$ effects, etc.)

- Natural or controlled current decay of the RE beam

- Average RE energy can be modified by timing or prescribed $U_{\text {loop }}$ waveforms

- Optimisation of position control algorithms

- Validation of models or elements of models that include the RE interaction with impurities (e.g. CODE [27], 28])

- Investigation of the RE transport in perturbed fields

- Exploitation of diagnostic methods under well controlled conditions

- Measurements of RE-wall or RE-limiter interactions during forced terminations

- Analysis of mutual interaction of RE with various instabilities

\section{Runaway electron beam position stability and analogies with plasma assisted modified betatron}

\subsection{Current control}

Current of the RE beam on COMPASS can be partly controlled in the case of the impurity injections using low amount of $\mathrm{Ar}$ or $\mathrm{Ne}$ (piezoelectric valve injection). However, the simultaneous control of radial position and beam current proved to be difficult. When the external loop voltage is removed, the beam current decreases with an average rate related to the type and amount of injected gas(es) [23] and the beam slowly drifts to the low field side. The application of a feedback control on the plasma current at values above $100 \mathrm{kA}$ requires loop voltage up to $2-4 \mathrm{~V}$ and the radial position is driven unstable. However, the beam current was successfully sustained when the current set-point was decreased, see figure 5, discharge \#14592, blue line. The current is typically related to the number of runaway electrons as the change in velocity is small for further accelerated relativistic electrons. Therefore, sustaining the RE beam current in the cold plasma background means namely to compensate the loss of particles by creating new RE. However, due to the high loop voltage, the energy of confined RE is further increased. This can be clearly seen in figure 5, where the external vertical magnetic field $B_{v}$ based on current flowing in the LFS poloidal coils [29] is indicated: despite constant beam current is maintained, the necessary vertical field to sustain the radial position is increasing up to very large values (over $100 \mathrm{mT}$ ). Moreover, the effect of ongoing increase of kinetic energy of RE occurs also in the case of spontaneously decaying current, e.g. in discharge \#16695 in figure 3, where zero external loop voltage was applied. The loop voltage induced by the current decay might not be sufficient 


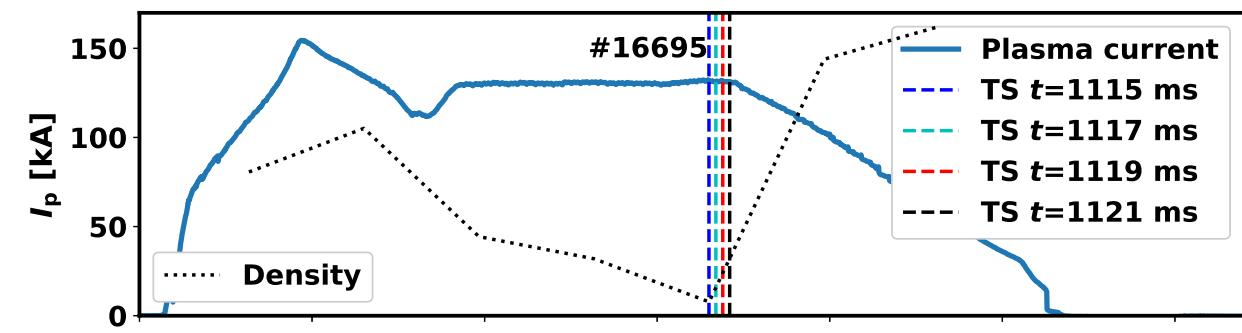

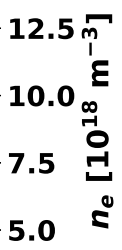
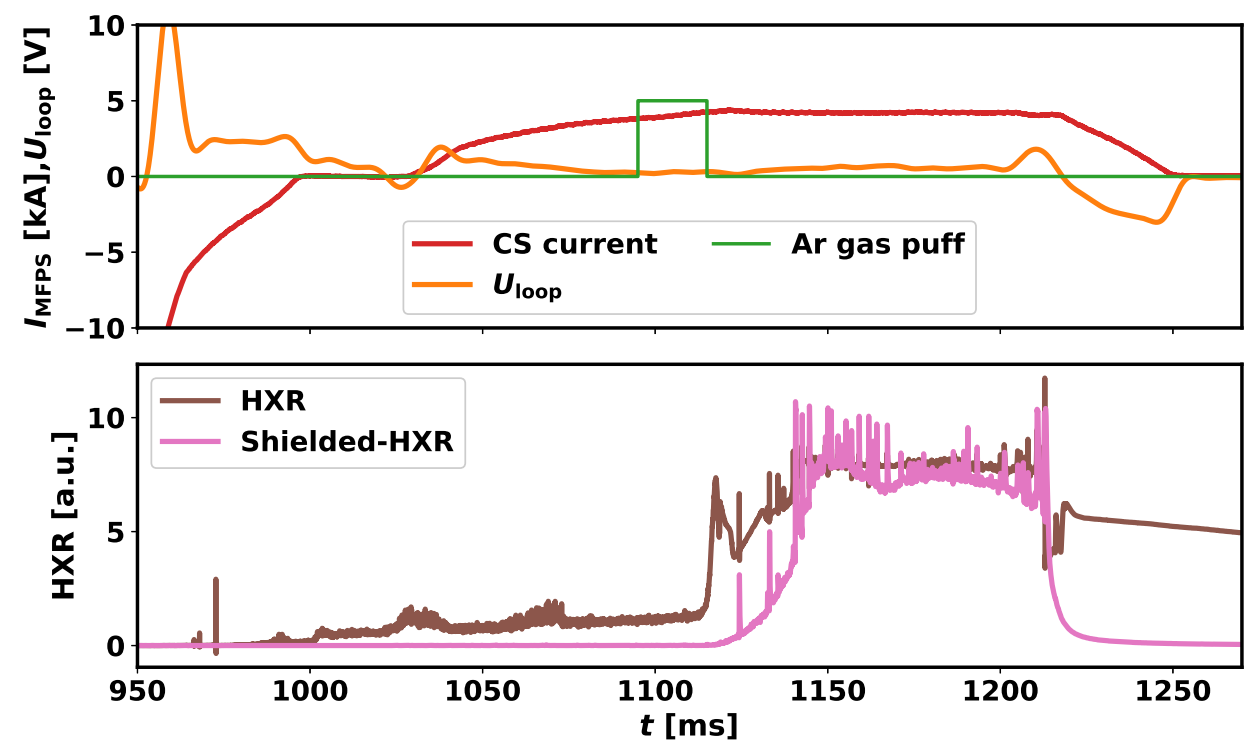

Figure 3. The flat-top scenario with RE beam generation - top: time evolution of plasma current in blue, electron desity measured by TS (black dotted line); center: loop voltage $U_{\text {loop }}$ in orange, current in the primary windings in red, Ar gas puff opening in green; bottom: HXR detector signals in brown $(>50 \mathrm{keV})$ and pink $(>500 \mathrm{keV})$.
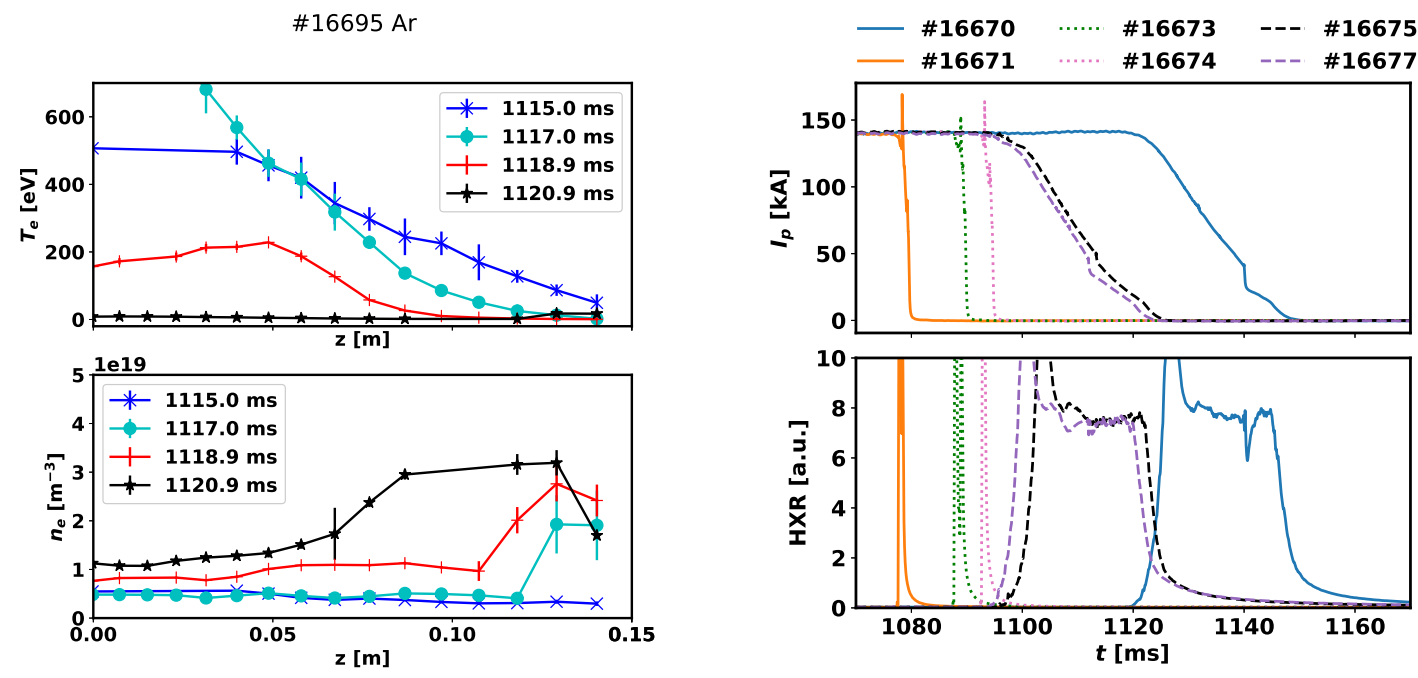

Figure 4. Left: The profiles of temperature and density from the TS diagnostics measured at times indicated in figure 3. Right: The timing scan with MGI Ar injection shows that the sufficient RE seed or sufficient RE energy is needed for creation and slow termination of a RE beam. 


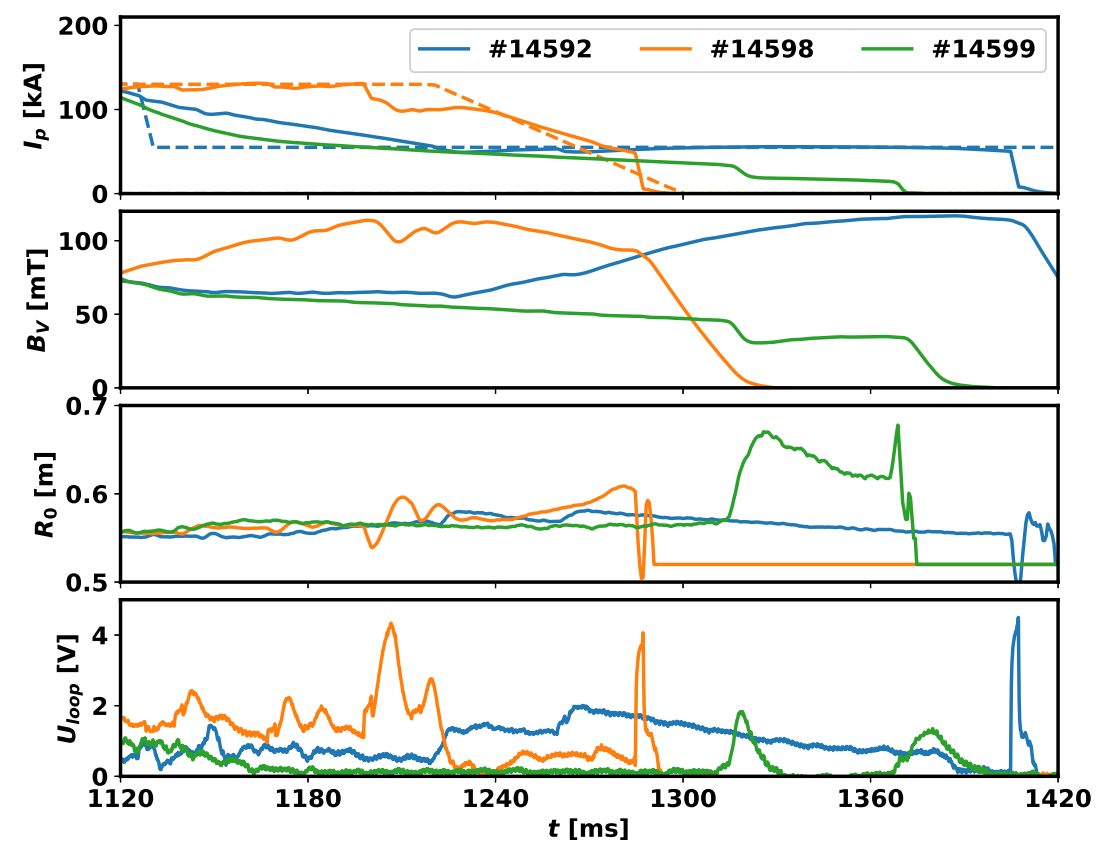

Figure 5. Different attempts to control the current of runaway electron beam, first frame: measured current and feedback reference (dashed) - in the case of discharge \#14599, zero loop voltage is requested; second frame: total external vertical field approximate value at $R_{0}$; third frame: Radial position of the plasma current centroid, bottom frame: loop voltage measured on the HFS. In the discharges \#14599 and \#14598, deuterium was injected during the RE beam phase (1120-1220 ms) [13] which led to a slower natural current decay.

for primary RE generation however it is sufficient for further acceleration of existing confined REs. Therefore, the position is unstable also during this type of the discharge.

\subsection{Radial stability of the relativistic electron beam and role of $R E$ energy}

The runaway orbits in equilibrium magnetic field and even the contribution of the runaway current to the total equilibrium were studied in many publications, including [30, [31]. At present devices, the radial position feedback policy during the RE beam stage is often modified based on empirical results and adaptive control, see [6], [32] as the physical model of this situation turns out to be rather complicated. The radial stability of the RE beam is incompatible with the standard feedback scheme applied on COMPASS and must be modified. The radial position on COMPASS is actuated by two different systems - equilibrium field power supply - EFPS and fast vertical magnetic field power supply - FABV [33]. The controller of the first one contains a term proportional to the plasma current (as a result of the Grad-Shafranov equilibrium, see eq. 2) and proportional-integral terms of the radial position error of the current centroid with respect to the reference. The FABV is dependent only on the position error and is also approximately five times weaker in maximum amplitude compared to FABV. The system performs excellently in case of a high temperature plasma without 
$\mathrm{RE}$, however the performance is poor with a $\mathrm{RE}$ beam in the low temperature plasma background as can be seen in figure 6. In the figure, various scenarios are compared in terms of plasma current evolution, normalised value of current in the EFPS windings $\left(I_{E F P S} /\left(7000+I_{p}\right)\right.$ - the constant is added so the function is stable when approaching zero - radial position and loop voltage which drives the current in plasma or accelerates the runaway electrons. It is obvious that while in the case displayed using green dotted line (standard discharge) the value of the function in the second frame is constant, in case of various RE scenarios: RE beams triggered by MGI (\#16635 - thick orange line) or piezoelectric valve Ar gas injection with plasma current feedback (\#14598 - violet line) or zero loop voltage applied (\#16625 - blue line, \#14599 - red dashed line + additional $\mathrm{D}$ injection added), the quantity is quickly increasing. The faster the current decay of the RE dominated plasma or loop voltage, the higher the request for the vertical field normalised by the plasma current value due to higher loop voltage induced during the current decay and subsequent increase in RE energy. Notice that despite large values of current in the stabilising windings the beam still drifts to the LFS. From this observation it is obvious that the radial feedback dependence on plasma current is too strong. The standard tokamak request for vertical field that results from Grad-Shafranov equation can be expressed as [34]

$$
2 \pi R_{0} I_{p} B_{v}=\frac{1}{2} I_{p}^{2} \frac{\partial}{\partial R_{0}}\left(L_{e}+L_{i}\right)-2 \pi^{2} \int d r r^{2} \frac{p^{\prime}-F F^{\prime}}{R_{0}^{2}},
$$

where the first term on the right hand side is related to external and internal part of "hoop force" (force between the current elements within the plasma ring), the second term (with $p^{\prime}$ ) to the tire "tube force" - expansion due to kinetic pressure gradient and the $F F^{\prime}$ term changes the direction with the value of $\beta_{p}$. The terms - except the $F F^{\prime}$ contribution - are always outward and typically depending on plasma current squared (the pressure term via the $\beta_{N}$ value). This means that vertical magnetic field should be also dependent on $I_{p}$. If we consider the case of the runaway electron beam - the hoop force terms should be still valid but the current density profile and therefore the internal inductance of the beam might be very different compared to thermal plasma. However, the gradient of classical thermal pressure is practically negligible for low temperature plasma background that may have even a large neutral fraction. Therefore, the dependence of vertical field on current in the beam ring should be weaker in the case of RE. Note that the size of the beam in terms of minor radius may also affect the feedback efficiency.

\subsection{Radial stabilisation of $R E$ beam}

A comparison of RE beams in tokamak with the high current betatrons - namely the modified ones (including the toroidal stabilising field) [35] - turns out to be appropriate. The average energy of beam of electrons plays a crucial role in the $B_{v}$ value necessary to keep the beam particles on radially stable orbits. If Larmor radius in the vertical magnetic field is considered (which applies in the case of a low beam current), the 


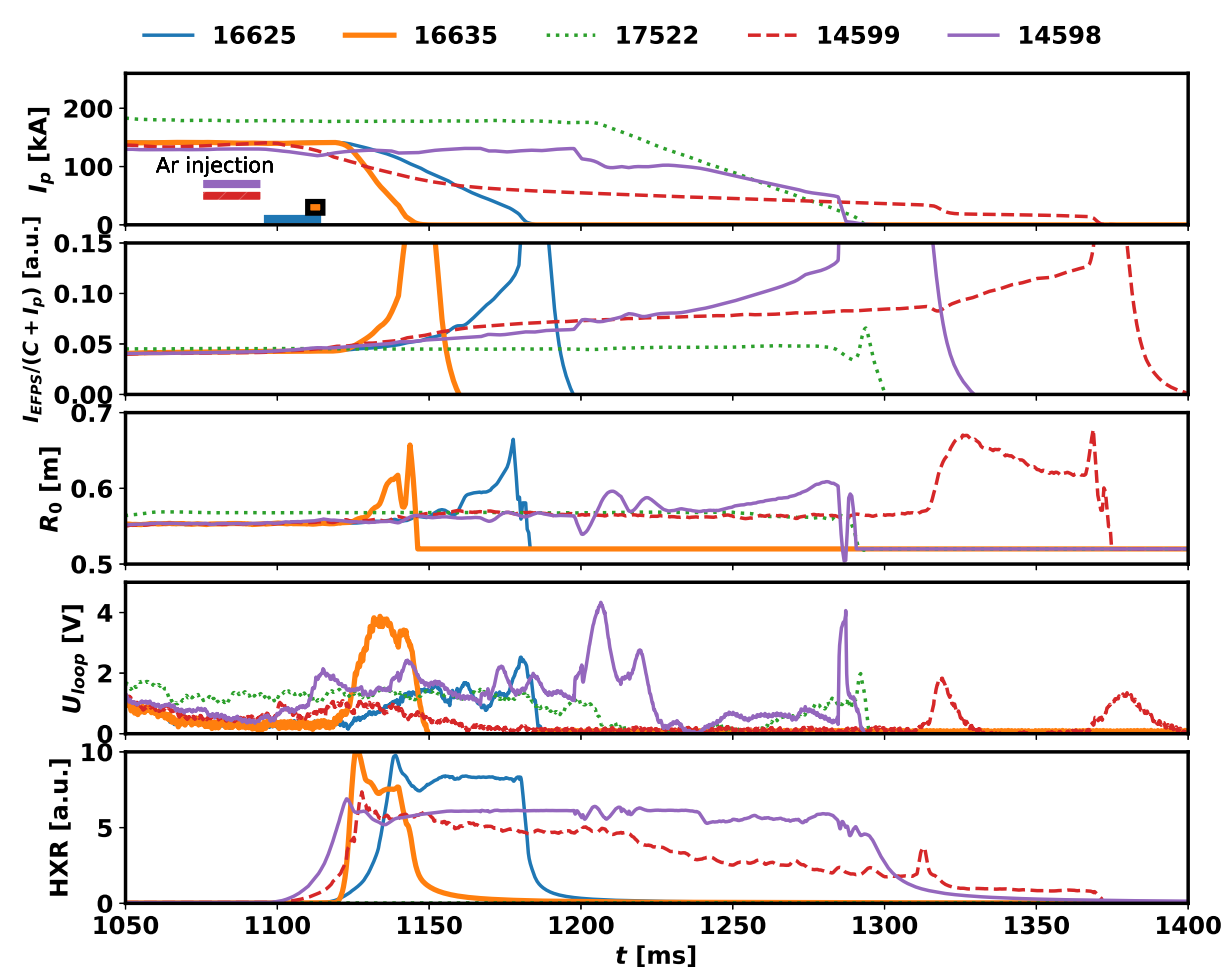

Figure 6. Comparison of radial control performance in the standard discharge (green) and various RE beam scenarios - Ar injection by MGI (\#16635), piezo valve injection (\#16625) or Ar injection followed by D injection (1120-1220 ms, \#14598, \#14599). The first frame shows the plasma current evolution and color coded rectangles marking the opening time of the gas injection valves, MGI is marked by a black frame. The second frame shows that the current requested from external poloidal field coils for radial position control is not a function of $I_{p}$ only in case of the RE beam. In the third frame, radial position evolution is displayed, the fourth frame shows the loop voltage values and the last one signal from the shielded HXR detector.

vertical field should be proportional to the flux change or average kinetic energy $\left\langle E_{k}\right\rangle$ of the accelerated electrons like in the classical betatron. A necessary value of the vertical field to confine a high current RE beam in the tokamak should be proportional to the mix of this contribution and the hoop force compensation:

$$
B_{v} \sim \frac{1}{4 \pi R_{0}} I_{p} \frac{\partial}{\partial R_{0}}\left(L_{e}+L_{i}\right)+\frac{\left\langle E_{k}\right\rangle}{\left(e c R_{0}\right)}
$$

in the ultra-relativistic case. Based on the theory for plasma-assisted modified betatrons, the beam in the plasma background should be always paramagnetic, unlike in the vacuum variant of the modified betatron, where diamagnetic to paramagnetic transition occurs during the acceleration of a high current beam according to conditions indicated in [35]. The paramagnetism is responsible for an additional confining force. In order to achieve a suitable feedback policy it is often sufficient to simply decrease the relative contribution of $I_{p}$-proportional part with respect to the contribution of radial position error term in the controller. Most of the tokamaks are close to the situation 
where $B_{v}$ - based primarily on $I_{p}$ - term is close to optimum for the given major radius and energies of RE in the range of tens of $\mathrm{MeV}$. On COMPASS, as a small device, the beam is typically drifting to the LFS in the quiescent stage, while overestimated vertical field may push the RE beam to the HFS or cause position oscillations in case of loss of some part of the current - see discharge \#14598 in figure 6. Standard feedback with decreased $I_{p}$ dependence may perform sufficiently well, but the equation 2 or a more complex model taking the average runaway electron energy into account should increase the efficiency of the feedback. On the other hand, the feedback algorithm disturbed in a controlled way, may be a source of information on the average energy of RE in the beam or even on the range of the energies. To investigate whether information on the flux change (electric field integral) is useful for estimating the optimal vertical field for radial position feedback, the discharge \#14592 can be further analysed. Let us assume that in the beginning of the $50 \mathrm{kA} \mathrm{RE}$ beam plateau (240 ms after the breakdown), the total requested vertical field is balancing well both the "hoop force" and the relativistic pressure arising from the change of energy. If only the second part of the equation 2 was taken into account (low current betatron approximation), the energy corresponding to the applied vertical field would be roughly $10-11 \mathrm{MeV}$ in the beginning of the constant current phase. This is in a reasonable agreement with other methods of determination of the upper energy limit for RE originating from the breakdown in various COMPASS discharges:

- Non-collimated HXR spectrometry shows that the energy limit of HXR reliably measurable with the 2" crystal $(7 \mathrm{MeV})$ is reached approximately after $150 \mathrm{~ms}$ of acceleration of RE in the COMPASS standard discharge with trace RE population. This measurement is unfortunately not available directly for the studied discharge due to extremely large HXR fluxes.

- Measurements of synchrotron radiation using mid-IR range camera $(15-25 \mu \mathrm{m})$ placed to a suitable tangential port show start of an increase of the measured power due to synchrotron radiation roughly $190 \mathrm{~ms}$ after the breakdown. This measurement is also an approximation as it is measured in a low-density discharge without gas injection. Based on the synchrotron radiation model SYRUP [36] using single energy approximation, only electrons with energy higher than 8-10 $\mathrm{MeV}$ give a non-negligible contribution in the camera spectral sensitivity range and the COMPASS magnetic field $\left(B_{t}=1-1.6 \mathrm{~T}\right)$.

- Last but not least, energy calculated using vacuum acceleration approximation based on loop voltage measured at the HFS reaches roughly $20 \mathrm{MeV}$ at the point of the start of the RE beam plateau in discharge \#14592. Vacuum approximation is providing overestimated values in general.

From the start of the constant current request phase, the beam current is stable, however the radial distribution of the current density might be changing and definitely there is a change in energy. In figure 7, it can be seen that the increase of applied vertical field is proportional to the electric field evolution through the relation marked 
in the legend of the graph, however a decreased value of the field derived from loop voltage measurement must be used in order to get a complete fit. This may result from the drag force due to impurities, imprecision of electric field measurement (loop external to the vessel) or other effects like change of the RE density profile and the RE energy distribution along minor radius.

\subsection{MHD equilibrium approximation for the RE beam}

The description mentioned above as a first approach is based on rather simplistic assumptions. A more suitable approach to the RE beam feedback is to use relativistic pressure. This was applied on the calculation of RE current fraction in thermal plasma in [37] and more recently in [38]. In this case, it is suitable to use relativistic pressure formula given by equation [37]

$$
p_{R E}=\frac{1}{2} m_{e}\left\langle n_{R E}\right\rangle\left\langle\gamma v_{\|}^{2}\right\rangle+\frac{1}{4} m_{e}\left\langle n_{R E}\right\rangle\left\langle\gamma v_{\perp}^{2}\right\rangle,
$$

where the RE density $\left\langle n_{R E}\right\rangle$ is averaged over beam volume and the velocity squared times Lorentz factor $\left\langle\gamma v_{\bullet}^{2}\right\rangle$ over both spatial and velocity distributions. Assuming that the relativistic pressure gradient is given primarily by the density gradient - rather than by the change of the average energy with the radius - the gradient of the RE pressure can be approximated by

$$
\begin{aligned}
\nabla p_{R E} & =m_{e} \nabla n_{R E}(r)\left(\frac{1}{2}\left\langle\gamma v_{\|}^{2}\right\rangle+\frac{1}{4}\left\langle\gamma v_{\perp}^{2}\right\rangle\right) \\
& \sim m_{e} \frac{\left\langle n_{R E}\right\rangle}{r_{b}}\left(\frac{1}{2}\left\langle\gamma v_{\|}^{2}\right\rangle+\frac{1}{4}\left\langle\gamma v_{\perp}^{2}\right\rangle\right),
\end{aligned}
$$

where $r_{b}$ is the beam minor radius. However, the effect of the radial profile of the average energy needs to be included as well to fully reconstruct the possible equilibria. The relativistic pressure gradient can be used in the MHD equilibrium $\nabla p=\mathbf{j} \times \mathbf{B}$ and modified equilibrium can be found. This introduces an additional component of vertical field. Measured electric field integral or electric field evolution as an output of a benchmarked disruption model seems to be a suitable input for physics-based

proportional controller of radial position in case of a relativistic beam. Such attitude may further increase performance of the well performing adaptive control algorithms currently run on the medium size devices. The relations will be tested on COMPASS during future RE experiments.

\subsection{Vertical stability}

To investigate the vertical stability of the RE beam created using the flattop recipe with Ar, a scan in the amplitude of the elongating field was carried out. It seems that the generation of the RE beam by a mitigation of the thermal plasma component via gas injection is not considerably affected by plasma elongation. As expected, the beam is 

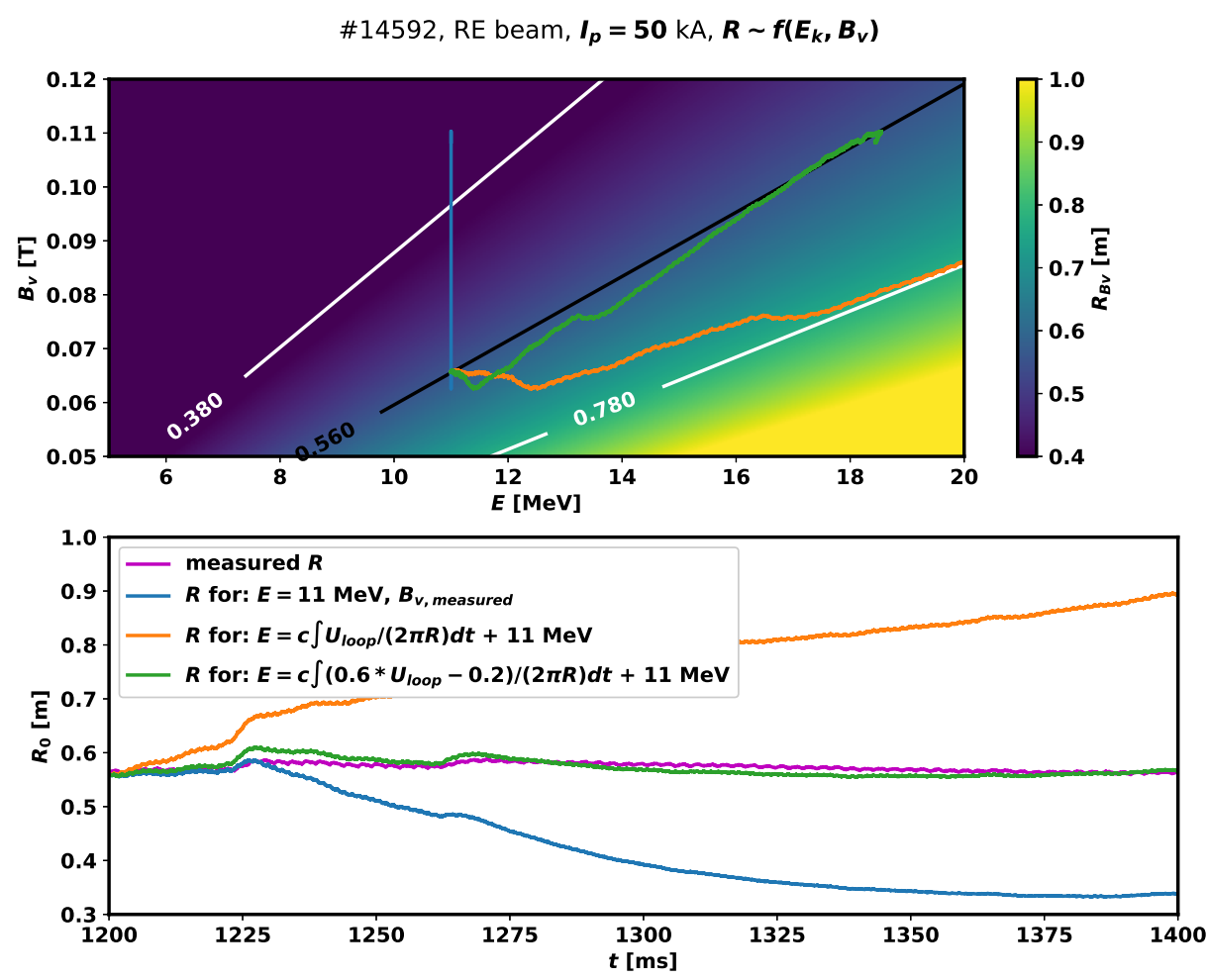

Figure 7. Top: 2D plane with ultra-relativistic electron Larmor radius as a function of energy and vertical field with curves of calculated $R_{B_{v}}$ for the signals of discharge \#14592; bottom: comparison of measured radial position signal and hypothetical position corresponding to $B_{v}$ evolution and energies obtained using different functions of electric field as marked in the legend.

prone to a vertical instability during the decay of its current and the instability occurs the earlier, the higher is the elongating field - this is reported in figure 8 . It seems that at the highest value of the elongation requested in the scan $(\kappa \sim 1.6)$ it is more difficult for the control system to sustain the current during the injection and slightly higher $U_{\text {loop }}$ is requested. The vertical displacement events last several ms and are characterised by large loop voltage spikes.

\section{Decay rate and energy loss channels}

The beam energy (magnetic and kinetic) can be lost through several channels - direct particle loss, radiation due to decay of excited atomic states of the background plasma species, induced currents in the vessel structures due to loss of the beam current, etc. Based on the gas amount scans with Ar and Ne, it seems that the two gases behave very differently in terms of the beam energy loss channels - while RE beam in Ne is radiating with larger power in the AXUV spectral region, Ar causes larger averaged signal of ${ }^{3} \mathrm{He}$ neutron counter and photomultiplier-based detector measuring high HXR fluxes during the beam decay while other HXR detectors are already saturated, see figure 9. Note that the ${ }^{3}$ He neutron counter can be also affected by the HXR if the fluxes are too large. 


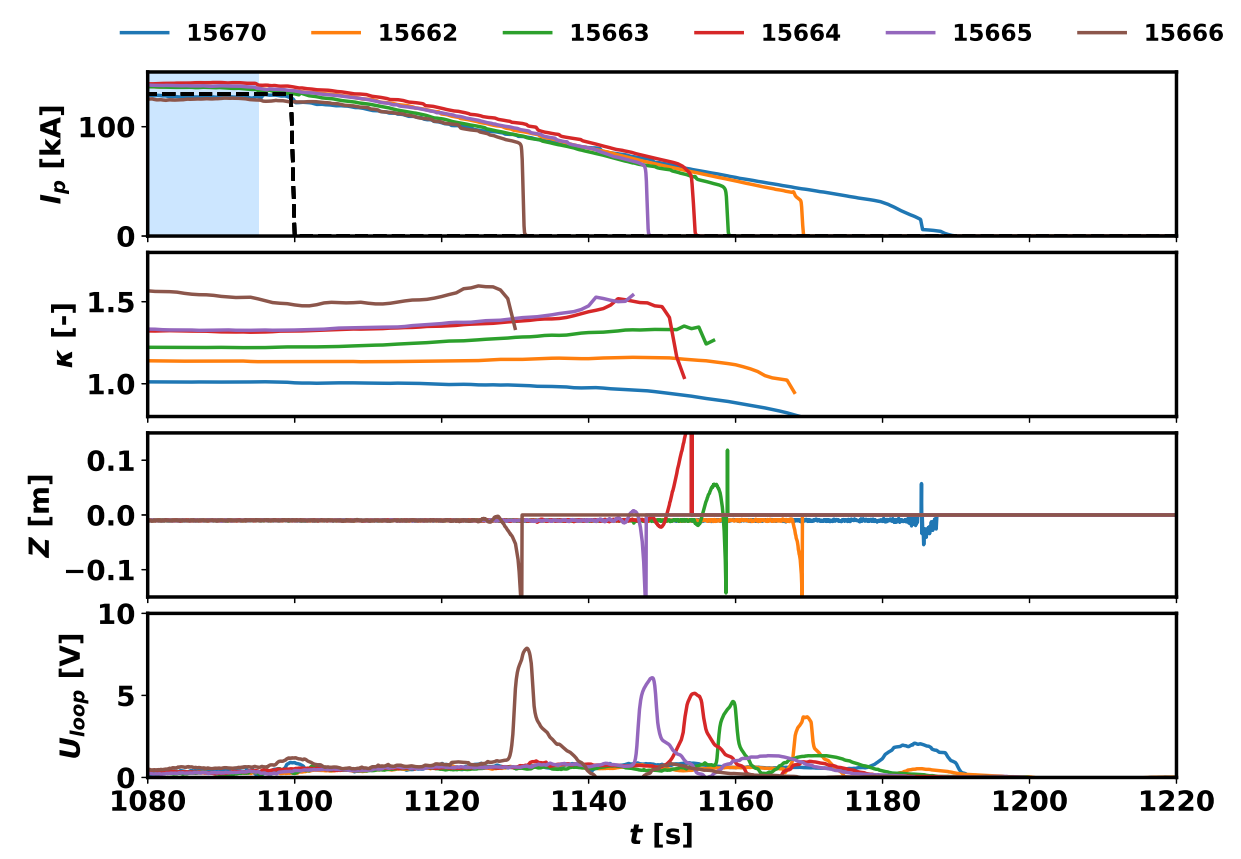

Figure 8. Elongation scan with piezoelectric gas puff Ar injection (1075-1095 ms): first frame - RE beam current evolution in zero external loop voltage regime (common reference - black dashed line, elongation increased with the discharge number); second frame - elongation as calculated by EFIT; third frame - measured vertical position; last frame: measured $U_{\text {loop }}$.

The radiated power was calculated by the modified tomography algorithm presented in section 1.3. More Ne is injected and higher pressure of the injected gas is measured if the same setup of valves is used for the given pressure - it is lighter and therefore moving faster both during the expansion into the vacuum and in the supplying tubes. However, the current decay rate of the RE beam is comparable for both gases with the same gas injection setup (see figure 3 in [13], discharges with various amount of injected Ar or $\mathrm{Ne}$ particles) and therefore the effect of the gases on the two energy loss channels can be directly compared. The relation may be a useful argument to prefer one gas over the other for different tasks. Argon seems to be a better choice to cause fast scattering of $\mathrm{RE}$ as a last layer of defence. On the other hand, if the RE beam position is stable and controlled, injecting large amounts of Ne can terminate the RE beam in a significantly more quiescent manner with large fraction of the energy being radiated in visible, UV and soft X-ray spectral regions.

\section{Conclusions}

Experiments using two different scenarios with gas injection triggered RE beam generation are under investigation at COMPASS: the ramp-up scenario and the flattop scenario. The former includes typical disruption features like current quench and is more relevant to larger machines in the terms of RE generation. However, the reproducibility 

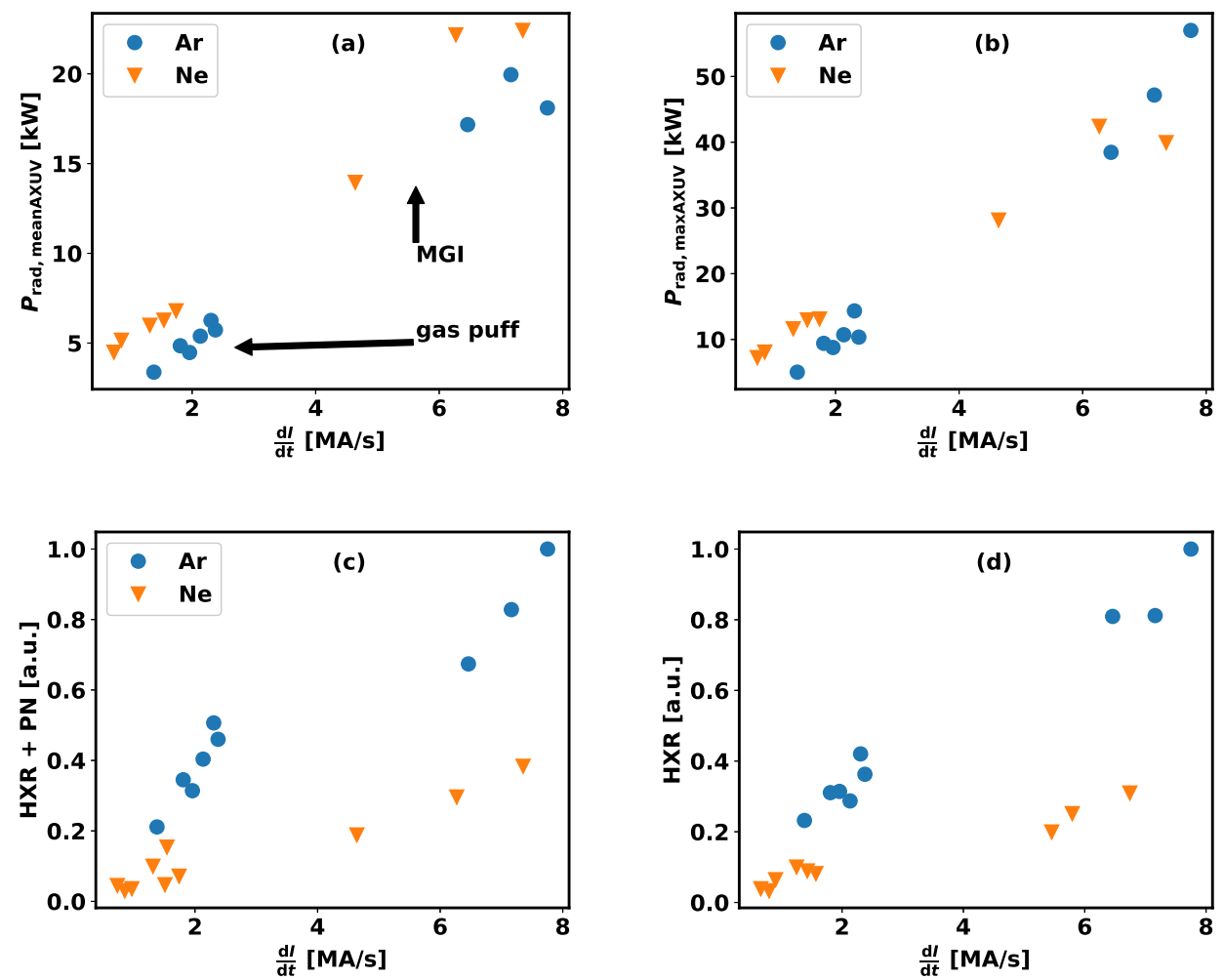

Figure 9. Gas amount scans for Ar and Ne, quantities plotted with respect to the average current decay rate of the RE beam: (a) the average radiated power during the beam decay calculated using AXUV tomography; (b) maximum AXUV radiated power during the beam decay; (c) number of counts of ${ }^{3} \mathrm{He}$ neutron detector (HXRs and photoneturons) normalised to beam decay duration; (d) average HXR as measured by blind photo-multiplier during the beam decay.

and control possibilities were not sufficient in this scenario on COMPASS. Therefore, a flattop scenario with high current $\mathrm{RE}$ beam was developed using various amounts of Ar or Ne to isolate the beam from the thermal plasma component. The current of the RE beam generated in this way may be kept at the desired value in the $\mathrm{Ar}$ or $\mathrm{Ne}$ background plasma at the cost of a relatively high loop voltage. However, it is even more interesting to switch off the external drive and observe the self-consistent decay of the beam. The radial position feedback was not performing very well possibly due to: (i) the absence of thermal pressure gradient that would require vertical magnetic field proportional to beam current and (ii) the role of RE energy missing in the request on the vertical magnetic field that would secure stable orbits. This hypothesis will be tested in the next campaigns. Regarding, the vertical position, the elongated RE beam seems to be stable even at relatively high values of elongation, unless the current decreases below a certain threshold. During the RE beam decay, some of the total energy is lost in the interaction with the gas (excitation, ionization and subsequent radiation) or directly through RE loss to the wall - Ar and Ne seem to behave oppositely. While Ar causes more high energy HXR signal indicating larger RE losses, Ne causes stronger 
radiation in the AXUV spectral region. The work on this scenario will further continue with the feedback optimization, puffing of gas mixtures, analysis of instabilities and investigation of magnetic field perturbation influence in order to provide a large set of reliable results to medium sized and large machines, where safety constraints are more limiting compared to COMPASS and to validate the key elements of runaway electron models.

\section{References}

[1] HENDER, T. C. et al., Nuclear Fusion 47 (2007) S128.

[2] BOOZER, A. H., Nuclear Fusion 57 (2017) 056018.

[3] LUKASH, V. et al., Study of ITER plasma position control during disruptions with formation of runaway electrons, in 40th EPS Conference on Plasma Physics, Aalto, Finland, 2013.

[4] SHIRAKI, D. et al., Shattered Pellet Injection as the Primary Disruption Mitigation Technique for ITER, in 26th IAEA Fusion Energy Conference, Kyoto, Japan, 2016.

[5] ESPOSITO, B. et al., Plasma Physics and Controlled Fusion 59 (2017) 014044.

[6] CARnEVAlE, D. et al., Plasma Physics and Controlled Fusion 61 (2019) 014036.

[7] SAINT-LAURENT, F. et al., Fusion Science and Technology 64 (2013) 711.

[8] PANEK, R. et al., Plasma Physics and Controlled Fusion 58 (2016) 014015.

[9] PANEK, R. et al., ITER-relevant research on the COMPASS tokamak, in 27th IAEA Fusion Energy Conference, Gandhinagar, India, 2018, IAEA.

[10] VLAINIC, M. et al., Journal of Plasma Physics 81 (2015) 475810506.

[11] PLYUSNIN, V. V. et al., Nuclear Fusion 58 (2018) 016014.

[12] FICKER, O. et al., Nuclear Fusion 57 (2017) 076002.

[13] MLYNAR, J. et al., Plasma Physics and Controlled Fusion 61 (2019) 014010.

[14] MACUSOVA, E. et al., The role of gas injection and magnetic perturbations in Runaway Electron experiments at COMPASS tokamak, in 60th Annual Meeting of the APS Division of Plasma Physics, Portland, Oregon, USA, 2018.

[15] GOBBIN, M. et al., Plasma Physics and Controlled Fusion 60 (2018) 014036.

[16] HAVLICEK, J. et al., Status of Magnetic Diagnostics on COMPASS, in WDS Proceedings of contributed papers, pages 12-17, Prague, Czech Republic, 2010, MATFYZPRES.

[17] BILKOVA, P. et al., Journal of Instrumentation 13 (2018) C01024.

[18] FARNIK, M. et al., Runaway electron diagnostic for the COMPASS tokamak using EC emissionn, in 45th EPS Conference on Plasma Physics, volume 2018, Prague, Czech Republic, 2018.

[19] RABINSKI, M. et al., Journal of Instrumentation 12 (2017) C10014.

[20] CEROVSKY, J. et al., Simulation of trajectories of runaway electrons for support of diagnostics at the COMPASS tokamak, in 45th EPS Conference on Plasma Physics, Prague, Czech Republic, 2018.

[21] WEINZETTL, V. et al., Journal of Instrumentation 12 (2017) C12015.

[22] MLYNAR, J. et al., Review of Scientific Instruments 83 (2012) 10E531.

[23] MLYNAR, J. et al., Journal of Fusion Energy (2018).

[24] ZENG, L. et al., Physical Review Letters 110 (2013) 235003.

[25] FICKER, O. et al., Analysis of MGI disruptions and runaway electron beams at COMPASS using tomography and fast camera data, in 45th EPS Conference on Plasma Physics, Prague, Czech Republic, 2018.

[26] FICKER, O. et al., RE beam generation in MGI disruptions on COMPASS, in 44th EPS Conference on Plasma Physics, Belfast, UK, 2017.

[27] STAHL, A. et al., Nuclear Fusion 56 (2016) 112009.

[28] HESSLOW, L. et al., Plasma Physics and Controlled Fusion 60 (2018) 074010. 
[29] HAVLICEK, J. et al., Characterization of Magnetic Fields in the COMPASS Tokamak, in WDS Proceedings of contributed papers, Prague, Czech Republic, 2008, MATFYZPRES.

[30] KNOEPFEL, H. et al., Nuclear Fusion 19 (1979) 785.

[31] YOSHIDA, Z., Nuclear Fusion 30 (1990) 317.

[32] HOLLMANN, E. M. et al., Nuclear Fusion 53 (2013) 083004.

[33] JANKY, F. et al., Fusion Engineering and Design 89 (2014) 186.

[34] FREIDBERG, J. P., Ideal MHD, Cambridge University Press, New York, revised edition, 2014.

[35] MANHEIMER, W. M., Part. Accel. 17 (1985) 157.

[36] STAHL, A. et al., Physics of Plasmas 20 (2013) 093302.

[37] FUJITA, T. et al., Journal of the Physical Society of Japan 60 (1991) 1237.

[38] VLAINIC, M. et al., Atoms 7 (2019) 12.

\section{Acknowledgments}

The work has been supported by the grant GA18-02482S of the Czech Science Foundation and also by the grant No. SGS19/180/OHK4/3T/14 of the Grant Agency of the Czech Technical University in Prague. The experiments were supported by projects No. CZ.02.1.01/0.0/0.0/16_019/0000768 and LM2015045, co-funded from European structural and investment funds and carried out within the framework of the EUROfusion Consortium. It has also received funding from the Euratom research and training programme 2014-2018 under grant agreement No 633053 with the Co-fund by the MEYS project number 8D15001. The views and opinions expressed herein do not necessarily reflect those of the European Commission. 